\title{
Productividad Laboral en Oficinas con uso de Sillas Basculantes
}

\author{
Jenny K. Rodríguez ${ }^{(1)}$, Fernanda Maradei(1)* y Javier Castellanos ${ }^{(2)}$ \\ (1) Universidad Industrial de Santander, Fac. de Ingeniería Físico Mecánicas, Escuela de Diseño Industrial, cra 27 \# 9 \\ Ciudad Universitaria, Bucaramanga-Colombia (e-mail: mafermar@uis.edu.co; jk.rodriguezgarcia@gmail.com) \\ (2) Universidad Pontifica Bolivariana. Facultad de Ingeniería Mecánica, Km 7 vía Piedecuesta, Bucaramanga, Colombia \\ (e-mail: javier.castellanos@upb.edu.co)
}

* Autor a quien debe ser dirigida la correspondencia

Recibido Nov. 19, 2018; Aceptado Ene. 16, 2019; Versión final Feb. 22, 2019, Publicado Ago. 2019

\begin{abstract}
Resumen
Se presenta evidencia que asocia la influencia de los asientos dinámicos con el tiempo productivo en trabajos de oficina. Veintiocho mujeres voluntarias participaron en el experimento que se realizó mediante el planteamiento de dos situaciones a) con basculación del asiento y b) sin basculación del asiento, en cuatro tareas de oficina: leer, escribir a mano, navegar en internet y digitar. En el estudio se midió el tiempo productivo y el número de errores. Se encontró que los asientos dinámicos no afectan significativamente el desempeño de los trabajadores. Por el contrario, los análisis descriptivos muestran mejores tiempos productivos y reducción en el número de errores. Se sugiere que la incorporación de asientos dinámicos debería ser un requerimiento importante en el diseño de sillas para oficina. Se ha demostrado que ellos disminuyen la incomodidad debida al dolor lumbar y contribuye a mejorar el rendimiento en el trabajo.
\end{abstract}

Palabras clave: dolor lumbar; ergonomía; silla; tiempo productivo; número de errores

\section{Office Productivity with the use of Tilt Motion Seats}

\begin{abstract}
Clear evidence that dynamic seats positively influences productivity is presented in this work. Twenty-eight voluntary women participated in an experiment involving two situations: a) with tilting seat motion and b) without tilting seat motion. Then, four common office tasks were assessed: reading, handwriting, internet browsing and keyboard writing. Two aspects were measured during the experiments: productive time and number of mistakes. It was found that a dynamic seat does not significantly affect office workers performance. On the contrary, the descriptive analysis shows higher productive time and lower number of mistakes. It is suggested that these dynamic seat configurations should be a requirement in the design of office seats. It has been shown that they reduce discomfort due to lumbar pain and contribute to increase work productivity.
\end{abstract}

Keywords: lumbar pain; ergonomic; seat; productive time; number of errors 


\section{INTRODUCCION}

Las demandas laborales del trabajo de oficina exigen mantener largas jornadas en postura sedente. Igualmente, la introducción intensiva de la informática ha modificado las actividades desarrolladas por los trabajadores y ahora deben permanecer en el puesto de trabajo de manera continua y en postura sedente prolongada (Karwowski y Marras, 1999), ocasionando la manifestación de diferentes trastornos musculoesqueléticos (TME). Diferentes estudios han podido establecer que existe una relación entre la postura sedente prolongada y el dolor lumbar (Lis et al., 2007). Los diagnósticos debidos a TME han aumentado en el ámbito nacional e internacional; el de mayor prevalencia a nivel mundial es el dolor lumbar (DL) (Collins y O'Sullivan, 2010). En el contexto colombiano, dicha patología es la segunda con mayor prevalencia en los trabajadores, interfiriendo en su desempeño laboral (Camargo et al., 2014). En este sentido diferentes autores han enfocado sus investigaciones a analizar y evaluar el trabajo de oficina, iniciando con la evaluación de la actividad laboral para encontrar las tareas con mayor penosidad, que afectan la salud del trabajador (Marklin y Wilzbacher, 1999; Ponsa et al., 2007), ya sea por la alta demanda física o por ser fisiológicamente exigentes. Específicamente en el trabajo de oficina, se ha encontrado que estas tareas son leer, digitar, escribir a mano y navegar en internet (Galindo et al., 2016).

Bajo este panorama, se han considerado intervenciones ergonómicas con asientos basculantes en conductores de vehículos pesados y en trabajo de oficina. Se ha encontrado que los cambios posturales del tronco pueden tener un efecto positivo en los procesos de nutrición biológica de la columna vertebral al reducir la carga estática de compresión sobre los discos (Grujicic et al., 2010). De forma similar, si se realizan ajustes en la rotación de la pelvis independientemente del tronco, tiene una influencia directa en la columna lumbar debido a que la pelvis forma la base de la curvatura lumbar (Van Geffen et al. 2010). Por tanto, se ha encontrado que el movimiento de la pelvis inducido por medio del asiento basculante, reduce la percepción de incomodidad debida al dolor lumbar cuando se está en postura sedente prolongada (Grujicic et al., 2010; Maradei et al. 2017; Van Geffen et al. 2010). Sin embargo, si bien la percepción de incomodidad debida al dolor lumbar se reduce, la revisión realizada en el marco de este proyecto no encontró evidencia que relacionen los diferentes tipos de tareas penosas de oficina con asientos basculantes, con el desempeño de los trabajadores. Se sabe que las posturas no neutrales adoptadas por los trabajadores de oficina favorecen la aparición de dolores, lo que conlleva a problemas de salud, tales como fatiga general, fatiga ocular, dolor de espalda y al aumento del tiempo no productivo, es decir al desempeño (Generalis y Mylonakis, 2007). Por consiguiente, la movilidad intervertebral inducida por el asiento debe producirse manteniendo rangos de amplitud que no comprometan las estructuras osteoligamentosas que dan estabilidad al conjunto del raquis para evitar futuros problemas de salud y de desempeño laboral.

Por otra parte, el trabajo de oficina al ser un tipo de trabajo de prestación de servicios, evidencia mayor dificultad en la medición de la productividad, pero se puede medir al igual que las actividades laborales de procesos de manufactura ya que el trabajo de oficina también es considerado como un proceso (Cabana et al. 2018). Estos procesos se pueden evaluar mediante estudios de tiempos asignándole suplementos. De esta manera, la evaluación por tiempos, definiendo tiempos productivos y no productivos, tienen la mayor aplicación para la evaluación ergonómica, buscando reducir las lesiones de los trabajadores sin detrimento del tiempo productivo (Resnick y Zanotti, 1997). De esta manera, este estudio busca responder a la pregunta: ¿En qué medida el uso de una silla con un sistema basculante en el asiento influye en el tiempo productivo de algunas tareas de oficina que son consideradas como penosas?. De esta forma, el objetivo fue determinar si existen diferencias significativas en el desempeño del trabajo de oficina, cuando se usa un asiento con basculación. Así, el estudio consideró que la basculación del asiento y el tipo de tarea que realizaban los participante, influían en las variables respuesta de tiempo productivo y el número de errores.

Esta investigación permitió encontrar nueva evidencia enfocada a la ergonomía laboral, tiempos productivos y promoción de la salud en el entorno laboral. De igual manera, mediante la asociación del tiempo productivo con las tareas de oficina y el tipo de uso del asiento, se esclarecen criterios ergonómicos para el diseño de asientos en la actividad de oficina. Se espera mejorar la calidad de vida de los trabajadores, asimismo estos hallazgos pueder ser aplicables al campo de la salud laboral, generando alternativas para la disminución de esta patología.

\section{MATERIALES Y MÉTODOS}

28 voluntarios, que hacían parte de la Universidad Industrial de Santander participaron en el experimento. Todos los participantes fueron mujeres entre los 21 y 45 años; es la población con mayor exposición a la postura sedente (Karlqvist et al. 1995). El criterio de exclusión considerado fue: (a) presentar dolor, adormecimiento o debilidad en la zona lumbar, manos, piernas y cuello, durante los últimos 12 meses, (b) haber realizado actividad física el día anterior al experimento, (c) haber tenido intervenciones quirúrgicas 
previamente y (d) presentar dolor antes o durante la prueba. El experimento tuvo el aval del Comité de Ética de la Universidad Industrial de Santander y por tanto todos los voluntarios firmaron el consentimiento para realizar las pruebas.

\section{Descripción de las Variables Independientes}

Factor (A) la silla a partir de 2 tratamientos: 1) Silla con asiento basculante (CB). Cuenta con un sistema en la parte inferior del asiento que permite tomar ángulos de movimiento hacia atrás de $10^{\circ}$ y hacia adelante 50, observado desde el plano sagital y 2) Silla sin basculación (SB), donde el asiento no puede inclinarse en ninguna dirección.

Factor (B) las tareas con mayor penosidad. Las tareas seleccionadas fueron: digitar, leer, escribir a mano y navegar en internet. Como generalidad y de acuerdo con los diferentes parámetros encontrados en la literatura, se plantearon tareas con una duración de 20 minutos cada una. La duración total de la experimentación por participante fue de 80 minutos por día durante dos días conservando la misma hora (Helander y Zhang, 1997).

\section{Tarea de digitar}

Se establecieron textos, para cada uno de los días de ejecución de la tarea, que tuvieran los textos en español (lengua nativa de los participantes), con citas de autores en inglés de reconocimiento a nivel mundial, sin tecnicismos, con narrador en primera persona contando sus experiencias y puntos de vista de manera clara y sencilla, con términos y tiempos verbales simples (considerando un nivel de escolaridad básica secundaria). De acuerdo con el estudio realizado por Werth y Babski-Reeves (2014), se proporcionó al participante el texto impreso, reduciendo al máximo la familiarización del participante con el material; teniendo una extensión de 600 palabras, dado que una persona adulta trascribe en promedio 30 palabras por minuto en texto copiado, mas no de memoria y con tipografía Arial 10 puntos (tamaño medio). De esta manera, en la Tabla 1 se muestra la caracterización de estas lecturas.

Tabla 1: Caracterización de las lecturas para la tarea digitar

\begin{tabular}{|l|l|}
\hline Lectura 1: El proceso de la innovación & Lectura 2: El mundo del futuro \\
\hline Total de palabras: 685 & Total de palabras: 685 \\
Tildes: 91 & Tildes: 91 \\
Comas: 27 & Comas: 27 \\
Punto seguido: 13 & Punto seguido: 12 \\
Punto aparte: 10 & Punto aparte: 11 \\
Palabras en inglés: 6 & Palabras en inglés: 7 \\
Símbolos: 5 & Símbolos: 5 \\
\hline
\end{tabular}

Al momento de ejecutar la prueba, el participante pudo ubicar el texto de acuerdo a la preferencia del sujeto, de forma natural. Este deberá trascribirlo en el programa de Microsoft office Word, versión 2013. Previamente se estableció en una prueba piloto un tiempo productivo 1379 segundos para esta actividad (Rodriguez et al. 2017). Para la identificación de los errores, se utilizó el estudio de Werth y Babski-Reeves (2014): (a)Inserta: definido como una letra extra que se inserta, en una palabra; (b) Omisión: cuando no se incluye una letra, en una palabra; (c) Cambian: cuando no se capitaliza una palabra o se capitaliza sin necesidad; (d) Sustitución: cuando se pone una letra errada en el lugar correcto de esa letra; (e)Trasponer: 2 letras consecutivas en una palabra son intercambiadas, (f) Uso de la tecla "suprimir" y "backspace".

\section{Tarea de Leer}

Se establecieron dos textos que tuvieran la particularidad de que los autores argumentan, de manera general, el tema de motivación laboral en el ambiente laboral. Además, con una extensión de 3600 palabras, estimada para ser leída en 20 minutos (Wainschenker et al. 2002) y las características mostradas en la Tabla 2. El participante desarrolló esta tarea leyendo en voz alta, para tomar grabaciones de audio, los archivos en formato PDF, letra sin serifa Arial 10 puntos. Además, el nivel del zoom era del $100 \%$ pero el participante lo podía cambiar a su conveniencia. El color de la tipografía era negro con fondo blanco para darle el máximo valor de contraste. El tiempo productivo de esta tarea es de 1195 segundos (Rodriguez et al. 2017) y se consideraron como errores, de acuerdo con el manual técnicas de lectura (Ugartemendia, 
2003), los siguientes: (a) Lectura de más de dos veces de una palabra, (b) Devolverse más de 2 veces en un párrafo, (c) Dejar de leer por más de 30 segundos (d) Regresiones: releer todo el contenido, (e) Subvocalización: releer en voz baja todo el contenido, (f) Cambiar palabras del texto.

Tabla 2: Caracterización de las lecturas para la tarea leer

\begin{tabular}{|c|c|}
\hline Lectura 1: El éxito de los perezosos & Lectura 2: El círculo de la motivación \\
\hline Número de palabras: 3672 & Número de palabras: 3668 \\
\hline Títulos: 6 & Títulos: 6 \\
\hline Puntos aparte:53 & Puntos aparte:46 \\
\hline
\end{tabular}

Tarea de escribir a mano

Perea y Perea (2014) establece que una persona en la etapa de educación secundaria alcanza progresivamente la escritura de 80 palabras por minuto. Tomando esto como dato base, se propone trascribir dos lecturas con aproximadamente 1600 palabras. Para este caso específico, se realizó una prueba piloto con 2 personas que contaban con el mismo grado de escolaridad, eran profesionales y con las edades límites del estudio ( 21 y 45 años), donde se determinó que los textos necesarios para escribir a mano debían tener menos de 500 palabras, para desarrollar la tarea en un tiempo mínimo de 20 minutos (Rodriguez et al. 2017).

La tarea se desarrolló en hojas de papel bond tamaño carta, sin cuadros ni líneas y un lapicero negro para que haya un mayor contraste. Los textos tenían las siguientes características mostradas en la Tabla 3. El tiempo productivo en esta tarea es de 1177 segundos (Rodriguez et al. 2017) y los errores se consideraron los mismos que en la tarea digitar.

Tabla 3: Caracterización del texto para transcribir a mano

\begin{tabular}{|c|c|}
\hline $\begin{array}{c}\text { Lectura 1. Manual de la comunicación } \\
\text { personal de éxito. }\end{array}$ & $\begin{array}{c}\text { Lectura 2. Comunicación interna } \\
\text { en la práctica. }\end{array}$ \\
\hline Número de palabras: 430 & Número de palabras: 430 \\
Tildes: 35 & Tildes: 39 \\
Comas: 23 & Comas: 20 \\
Puntos seguidos: 12 & Puntos seguidos: 10 \\
Símbolos: 13 & Símbolos: 15 \\
\hline
\end{tabular}

\section{Tarea de navegar en internet}

Para el planteamiento de esta tarea, se determinaron las características de una página web con contenidos jerarquizados, sistema de búsqueda, opciones de navegación clara, buena relación figura-fondo y que tuvieran una tipografía adecuada; ya que los estudios encontrados están relacionados con la evaluación de las características gráficas de las páginas web, mas no de una tarea específica para realizar esta. De esta manera, se propuso la tarea de navegar en internet con cada uno de los pasos a realizar, ejecutada con 140 clicks, la concentración de la información en los mismos espacios, la tarea de crear un correo electrónico y un carrito de compras, y el tiempo teórico de ejecución de 20 minutos (Tabla 4).

El tiempo productivo de esta tarea es de 1360 segundos (Rodriguez et al. 2017), se tomaron video grabaciones de la pantalla y los errores fueron definidos como: 1) No agregar alguno de los productos especificados en la Tabla 4 y 2) Después de los 168 clics, cada clic será considerado como un error. En este caso se especifica que el número de clics total para desarrollar la tarea es de 140 y se consideran 28 clics (20\% del total de clics) como posible margen de error.

\section{Descripción de las variables dependientes}

El tiempo productivo en la ejecución de cada tarea. Para esto, fue necesario ejecutar una prueba piloto para establecer el tiempo total trabajado en consideración a cada una de las tareas planteadas, comparando entre los participantes de esta prueba el ritmo en la ejecución del trabajo con lo que se considera normal, considerando los suplementos (necesidades personales, condiciones atmosféricas, concentración intensa, ruido, tensión mental, monotonía y tedio). Esta prueba piloto fue publicada en la Revista UIS ingenierías en 
el 2017 (Rodriguez et al. 2017). Los errores en la ejecución de cada tarea, fueron medidos de acuerdo con cada una de las particularidades de la tarea a desarrollar descritas en el ítem anterior.

Procedimiento

La ejecución del experimento se inició con la explicación a cada uno de los participantes del objetivo del estudio, el acercamiento a cada uno de los equipos encargados de la toma de datos, la lectura y firma del consentimiento informado y el registro de los datos de cada participante. Seguido a esto, se asignó aleatoriamente los tratamientos, para cada uno de los participantes, reduciendo el sesgo en la toma de los datos.

Tabla 4: Pasos para la tarea de navegar en internet

\begin{tabular}{|c|c|}
\hline Día 1 & Día 2 \\
\hline 1. Crear correo electrónico en www.gmail.com: & 1. Crear correo electrónico en www.hotmail.com \\
\hline $\begin{array}{l}\text { Registrarse con el correo creado en la página } \\
\text { www.exito.com }\end{array}$ & $\begin{array}{l}\text { Registrarse con el correo creado en la página } \\
\text { www.falabella.com.co }\end{array}$ \\
\hline \multicolumn{2}{|l|}{ 2. Comprar los siguientes artículos: } \\
\hline \multicolumn{2}{|c|}{$\begin{array}{l}1 \text { televisor de } 43 \text { ": Marca LG, ordenar los precios de menor a mayor, agregar al carrito el de menor valor y eliminar } \\
\text { la garantía. }\end{array}$} \\
\hline \multicolumn{2}{|c|}{$\begin{array}{l}1 \text { televisor de } 32 \text { " Cualquier marca, ordenar por precio de menor a mayor, agregar al carrito el más económico y } \\
\text { eliminar la garantía. }\end{array}$} \\
\hline \multicolumn{2}{|c|}{1 nevera minibar marca HACEB, ordenar de mayor a menor, añadir al carrito la de mayor valor. } \\
\hline \multicolumn{2}{|c|}{$\begin{array}{l}1 \text { cafetera marca black and decker, añadir al carrito la } 2^{\circ} \text { de menor precio y con garantía por } 1 \text { año o por defecto la } \\
\text { que trae la página. }\end{array}$} \\
\hline \multicolumn{2}{|c|}{$\begin{array}{l}1 \text { aire acondicionado que el precio oscile entre } 1 \text { y } 2 \text { millones de pesos, ordenar por los más recomendados, añadir } \\
\text { al carrito la referencia que se encuentra en la posición 12, con garantía de } 1 \text { año o la que por defecto da la página } \\
\text { de internet. }\end{array}$} \\
\hline \multicolumn{2}{|l|}{3 escritorios del menor valor, sin garantía. } \\
\hline \multicolumn{2}{|c|}{$\begin{array}{l}9 \text { sillas de oficina del mayor valor con garantía por } 1 \text { año o la que por defecto da la página de internet. Todas las } \\
\text { sillas deben ser de la misma referencia. Si no hay en stock las cantidades solicitadas, seleccionar la siguiente con } \\
\text { las mismas características. }\end{array}$} \\
\hline \multicolumn{2}{|l|}{1 biblioteca del menor valor. } \\
\hline \multicolumn{2}{|c|}{1 juego de sala para exteriores, la primera de 4 puestos que ofrezca la página. } \\
\hline \multicolumn{2}{|l|}{1 tapete decorativo color gris. } \\
\hline
\end{tabular}

Finalmente, se le indicó al participante que se sentara para ajustar las condiciones del asiento respecto al puesto de trabajo. De esta manera, se dio inicio a la toma de datos desarrollando las 4 tareas asignadas, sin tiempo limitante en la ejecución de cada una. Entre cada tarea, se determinó una pausa, realizando un ciclo de ejercicios espinales de 25 segundos, que permitirían relajar la espalda. El participante al momento de la ejecución de las tareas establecidas se encontraba dentro de un laboratorio, en el cual podía modificar la temperatura del aire acondicionado, las herramientas del puesto de trabajo y desenvolverse con naturalidad ya que nadie más se encontraba dentro de este espacio. Por otro lado, el investigador encargado y el auxiliar del grupo de investigación tomaba los datos y observaba al participante por medio de la cámara de Gesell, sin interferir ni interrumpir al participante.

\section{Análisis estadístico}

Los tiempos productivos (segundos) y el número de errores se analizaron por cada tratamiento asignado. Para identificar las diferencias significativas se realizó el análisis de varianza ANOVA, ya que los datos fueron paramétricos, donde el nivel de significación fijado fue de $5 \%$, considerando valores estadísticamente significativos cuando $p$-valor $\leq 0,05$.

\section{RESULTADOS}

Para la toma de datos se convocó a todas las trabajadoras de la UIS, directamente en sus puestos de trabajo, con el visto de sus jefes directos. 36 participantes accedieron a realizar la prueba, de las cuales 2 no asistieron el segundo día de la prueba, 1 fue excluida por superar el límite de edad planteada para la prueba y 5 no accedieron a programar las citas para ejecutar la prueba. Así el experimento fue realizado con 28 participantes, buscando una muestra homogénea para lograr mejores resultados en las 
comparaciones de medias. La Tabla 5 describe a la muestra estudiada, teniendo en promedio 25 años, $56,28 \mathrm{Kg}$ de peso, $1,60 \mathrm{~m}$ de altura y 21,8 IMC. Asimismo, el 57,1\% de la población participante fueron trabajadoras administrativas, 32,2\% auxiliares administrativas o de investigación, 3,57\% profesoras y el $7,13 \%$ estudiantes en proyecto de grado de pregrado y maestría.

Tabla 5: Descripción de los participantes

\begin{tabular}{|c|c|c|c|c|}
\hline & Mínimo & Máximo & Promedio & DS \\
\hline Edad (años) & 21 & 45 & 25,75 & 5,15 \\
\hline Peso $(\mathrm{Kg})$ & 47 & 74 & 56,28 & 6,84 \\
\hline Altura $(\mathrm{m})$ & 1,5 & 1,78 & 1,6 & 0,06 \\
\hline IMC & 17,35 & 27,85 & 21,89 & 2,55 \\
\hline
\end{tabular}

\section{Tiempo productivo y número de errores}

El análisis descriptivo muestra que Navegar en Internet en el asiento CB tiene mayor tiempo productivo ya que gasta $19 \%$ de tiempo adicional con relación al asiento SB y tiene un 30\% menos de errores. En la tarea leer y digitar en el asiento $\mathrm{CB}$, se tarda un poco más en hacer la tarea, pero con menor número de errores. En la tarea de escribir a mano, el tiempo con los dos tipos de asientos tuvieron valores muy cercanos, casi iguales (ver tabla 6).

Por otro lado, los errores al desarrollar las tareas en el asiento CB fueron menores en las tareas digitar, navegar en internet y leer. También mediante la comparación de medias a través de ANOVA, se evidenció que no hay diferencias significativas en el tiempo productivo $(p=0,585)$ y el número de errores $(p=0,573)$. En este último aspecto, también se consideraron el número de clicks promedio al desarrollar la tarea navegar en internet, encontrando que al realizar esta tarea en el asiento CB es menor (número de clicks: 193,03) comparado con el asiento SB (número de clicks: 210,75). Los valores en cada casilla en la Tabla 6 corresponden al tiempo adicional al $100 \%$ del tiempo productivo estandarizado en segundos (TE).

Tabla 6: Tiempos productivos y errores de las 4 tareas en cada tratamiento

\begin{tabular}{|c|c|c|c|c|c|}
\hline \multirow{2}{*}{ Tarea } & \multicolumn{2}{|c|}{ CB } & \multicolumn{2}{c|}{ SB } & TE \\
\cline { 2 - 5 } & tiempo & errores & tiempo & errores & $\begin{array}{c}\text { Tiempo estandarizado } \\
(s)\end{array}$ \\
\hline Digitar & $+13,81 \%$ & 86,071 & $+11,87 \%$ & 94,21 & 1379 \\
\hline Escribir a mano & $+11,68 \%$ & 12,21 & $+11,44 \%$ & 10,10 & 1177 \\
\hline Navegar en internet & $+19,29 \%$ & 2,821 & $+30,74 \%$ & 4,107 & 1361 \\
\hline Leer & $+13,47 \%$ & 12,39 & $+12,68 \%$ & 17,1 & 1195 \\
\hline Promedio & $+14,562 \%$ & 28,37 & $+16,68 \%$ & 31,37 & 1278 \\
\hline
\end{tabular}

\section{DISCUSIÓN}

De acuerdo con la pregunta de investigación del estudio ¿En qué medida el uso de una silla con un sistema basculante en el asiento influye en el tiempo productivo de algunas tareas de oficina que son consideradas como penosas?, se pudo determinar que, aunque no existen diferencias significativas entre el tipo de asiento en el tiempo productivo y el número de errores, en el asiento CB las tareas se ejecutaron en promedio con un tiempo menor y con un menor número de errores en comparación con la silla $\mathrm{SB}$. Esto puede darse debido a que el diseño basado en principios ergonómicos puede eliminar dificultades a la hora de realizar el trabajo y conducir a reducir el tiempo improductivo, lo cual, a largo plazo maximiza el desempeño y la rentabilidad de las organizaciones (Generalis y Mylonakis, 2007; Guasch et al., 2004). Los resultados de estudios donde se realizaron modificaciones de los puestos de trabajo de oficina reduciendo las posturas estáticas, muestran resultados similares a este estudio en términos de tiempos de ejecución de las tareas y número de errores (Elmer y Martin, 2014; Koren et al., 2016), asimismo además de la productividad laboral se ha evidenciado una mejora en la satisfacción laboral (Buman et al., 2017).

Es fundamental resaltar la incidencia de otros factores sobre el desempeño, como la experiencia del trabajador, el trabajo en equipo, factores psicosociales, y la carga del trabajo que conducen al mejoramiento del rendimiento personal (Kazmierczak et al., 2007). En términos de comportamiento postural, se ha encontrado que estar sentado en una silla dinámica reduce la flexión lumbar (O'Sullivan et al., 2012), lo que 
sugiere una postura lumbar más natural y disminución de la incomodidad, esto explicaría la influencia positiva del asiento CB en el número de errores de las tareas de oficina estudiadas.

En este sentido, con relación al número de errores (calidad de ejecución de las tareas), se encontró que estos son siempre menores en todas las actividades con el computador realizadas en el asiento CB: digitar, navegar en internet y leer, aumentando la calidad y productividad del trabajo (Yeowa y Sen, 2006). Solo se obtuvo en la tarea escribir a mano un mayor número de errores, generado posiblemente por la fatiga, lo cual disminuye la velocidad y calidad en el trabajo (Resnick y Zanotti, 1997), sin embargo, no se encontraron diferencias estadísticamente.

Así el comportamiento postural hace que las tareas que requieren atención, exactitud y la necesidad de ejecutar entrada de datos a gran velocidad en sistemas informáticos, mantienen una inclinación del tronco hacia adelante, en la medida que se requiera ejecutar correctamente (Galindo et al., 2016), con un menor dinamismo en la espina cervical y torácica (Ohlinger et al., 2011). Por esa razón, se puede entender que, el asiento CB el cual tiene un efecto que favorece la reducción de dichos ángulos lumbares genere también una postura dinámica en constante cambio, manteniendo al raquis entre los rangos de movimiento permitidos y favoreciendo la nutrición discal. El coeficiente de fricción sobre el asiento es uno de los factores que más influyen en la carga intradiscal, por tanto algunos estudios sugieren que las inclinaciones hacia adelante no deben superar los $10^{\circ}$ (Rasmussen et al., 2009).

Con base en esto, la evaluación del efecto de una silla dinámica en el tiempo productivo muestra que la basculación del asiento además de mitigar la percepción de incomodidad debida al dolor, evidenciado ya por múltiples artículos (Maradei et al., 2017; O'Sullivan et al., 2012; Van Geffen et al., 2010), no afecta significativamente el desempeño de los trabajadores, por el contrario, los análisis descriptivos muestran mejores tiempos productivos y reducción el número de errores. Por tanto, se sugiere que este debería ser un requerimiento importante en el diseño de sillas para oficinas, dado que la evidencia muestra que al reducir los riesgos biomecánicos se contribuye a mejorar el rendimiento humano (Generalis y Mylonakis, 2007; Resnick y Zanotti, 1997). Por último, así como las consideraciones estéticas en las sillas son requerimientos importantes en el diseño, también lo son las consideraciones ergonómicas que pueden permitir mejorar la percepción de incomodidad a partir de la reducción de las demandas biomecánicas en el raquis.

Por otra parte, el estudio identificó de forma observacional que las personas ajustaron las sillas SB al iniciar cada una de las tareas, pero al usar el asiento CB tenían una actitud de rechazo hacia éste debido a la incompatibilidad con el modelo mental de uso o a lo no conocido. Esto conduce a afirmar que al cambiar el entorno del puesto de trabajo debe darse un acompañamiento continuo con retroalimentación constante (Galindo et al. 2016). Por último, se sugiere, realizar estudios con población masculina y con personas con dolor lumbar, que ampliarían los resultados obtenidos de esta investigación.

\section{CONCLUSIONES}

Con base en los hallazgos más importantes del estudio, se puede concluir que 1) en los asientos dínamicos, donde existe una basculación del asiento, el desempeño de los trabajadores de oficina es mejor en comparación con los asientos estáticos. 2) Se evidencia mejores resultados en el tiempo productivo y 3) una reducción del número de errores al ejecutar tareas como digitar, navegar en internet o leer.

\section{REFERENCIAS}

Buman, M.P., S.L. Mullane y otros 8 autores, An Intervention to Reduce Sitting and Increase Light-Intensity Physical Activity at Work: Design and Rationale of the 'Stand \& Move at Work' Group Randomized Trial, doi: 10.1016/j.cct.2016.12.008, Contemp Clin Trials, 53, 11-19 (2017)

Cabana, S.R., H.J. Narea y R.D. Orrego, Factores Determinantes de la Conducta Intraemprendedora en Pequeñas y Medianas Empresas (PyMes) de la Región Coquimbo en Chile, http://dx.doi.org/10.4067/S0718-07642018000400167, Inf. Tecnol, 29(4), 167-178 (2018).

Camargo, D., J. Jimenez, E. Archila y M. Villamizar, El Dolor: Una Perspectiva Epidemiológica, https://revistas.uis.edu.co/index.php/revistasaluduis, ISSN: 2145-8464, Salud UIS, 36(1), 2-13 (2014)

Collins, J. y L. O'Sullivan, Phychosocial Risk Exposures and Musculoskeletal Disorders Across Working-age Males and Females, https://doi.org/10.1002/hfm.20220, Human Factors and Ergonomics in Manuf. \& Service Industries, 20, 272$286(2010)$

Delgado, A.C., F. Maradei y J. Castellanos, Influencia de los Patrones Posturales en la Conducción y la Antropometría en la Carga Biomecánica del Raquis, https://revistas.upb.edu.co/index.php/iconofacto/index, ISSN: 2390-0040, Iconofacto, 9(12), 38-55 (2013) 
Elmer, S. y J. Martin, A Cycling Workstation to Facilitate Physical Activity in Office Settings, https://doi.org/10.1016/j.apergo.2014.03.001, Appl Ergon, 45(4),1240-1246 (2014)

Galindo, Z., F. Maradei y F. Espinel, Percepción del Dolor Lumbar Debido al Uso de un Asiento Dinámico en Postura Sedente Prolongada, doi: https://doi.org/10.15446/rsap.v18n3.42897, Rev. Salud Pública, 18(3), $412-424$ (2016)

Generalis, G. y J. Mylonakis, Productivity and Ergonomics: A Strong Relationship Leading to Best Working Results, https://www.europeanjournalofsocialsciences.com, ISSN: 1450-2267, Eur. J. Soc. Sci., 5(1), 54-60 (2007)

Grujicic, M., B. Pandurangan y otros 4 autores, Musculoskeletal Computational Analysis of the Influence of Car-seat Design/adjustments on Long-distance Driving Fatigue, https://doi.org/10.1016/j.ergon.2010.01.002, Int. J. Ergon, 40(3), 345-355 (2010)

Guasch, A., M.A. Piera, J. Casanovas y J. Figueras, Modelado y Simulación. Aplicación a Procesos Logísticos de Fabricación y Servicios, Ediciones UPC (2004)

Helander, M.G. y L. Zhang, Field Studies of Comfort and Discomfort in Sitting, https://doi.org/10.1080/001401397187739, Ergonomics, 40(9), 895-915 (1997)

Karlqvist, L., M. Hagberg, G.A. Hansson y M. Waldenström, Good Working Environments for Graphics Industry VDU Operators Using a Mouse, Second International Scientific Conference on Prevention of Work-related Musculoskeletal Disorders, Montreal, Canada, 24-27 de septiembre (1995)

Karwowski, W. y W. Marras, The Occupational Ergonomics Hand Book, $1^{\text {a }}$ Ed., CRC Press, EE UU (1999)

Kazmierczak, K., W. Neumann y J. Winkel. A Case Study of Serial-Flow Car Disassembly: Ergonomics, Productivity and Potential System Performance, https://doi.org/10.1002/hfm.20078, Hum Factors Ergon Manuf, 17(4), 331 -351 (2007)

Koren, K., R. Pisot y B. Simunic, Active Workstation Allows Office Workers to Work Efficiently While Sitting and Exercising Moderately, doi: 10.1016/j.apergo.2015.11.013, Appl Ergon, 54, 83-89 (2016)

Lis, A.M., K. M. Black, H. Korn y M. Nordin, Association Between Sitting and Occupational LBP, doi: 10.1007/s00586006-0143-7, Eur Spine J., 16(2), 283-298 (2007)

Maradei, F., L. Quintana y J. Castellanos, Influence of Seat Tilt Motion on Discomfort Perception During a Simulated Driving Activity, doi: 10.3233/WOR-162464, Work. 56(1), 99-110 (2017)

Marklin, R.W. y J. Wilzbacher, Four Assessment Tools of Ergonomics Interventions: Case Study at an Electric Utility's Warehouse System, https://doi.org/10.1080/00028899908984501, Am Ind Hyg Assoc J, 60(6), 777-784 (1999)

Ohlinger, C.M., T.S. Horn, W.P. Berg y R. Cox, The Effect of Active Workstation Use on Measures of Cognition, Attention, and Motor Skill, https://doi.org/10.1123/jpah.8.1.119, J. Phys. Act. Health, 8(1), 119-125 (2011)

O'Sullivan, K., R. McCarthy y otros tres autores, Lumbar Posture and Trunk Muscle Activation During a Typing Task When Sitting on a Novel Dynamic Ergonomic Chair, doi: 10.1080/00140139.2012.721521, Ergonomics, 55(12), 15861595 (2012)

Perea, A. y M. Perea, Desarrollo Didáctico de la Escritura, https://revistascientificas.us.es/index.php/CAUCE, ISSN: 0212- 0410, CAUSE Revista Internacional de Filología, Comunicación y sus Didácticas, (36), 237-250 (2013)

Ponsa, P., R. Vilanova y M. Díaz, Introducción del Operario Humano en el Ciclo de Automatización de Procesos Mediante la Guía GEMMA, http://dx.doi.org/10.4067/S0718-07642007000500004, Inf. Tecnol, 18(5), 21-30 (2007)

Rasmussen, J., S. Torholm y M. de Zee, Computational Analysis of the Influence of Seat Pan Inclination and Friction on Muscle Activity and Spinal Joint Forces, https://doi.org/10.1016/j.ergon.2008.07.008, Int. J. Ind. Ergon, 39(1), 52-57 (2009)

Resnick, M.L. y A. Zanotti, Using Ergonomics to Target Productivity Improvements, https://doi.org/10.1016/S03608352(97)00070-3, Computers ind. Engng, 33(1),185-188 (1997)

Rodriguez, J., F. Maradei y J. Castellanos, Effect of Seat Tilt Motion in The Productive Time: Pilot Study, https://revistas.uis.edu.co/index.php/revistauisingenierias, ISSN: 2145-8456, Revista uis ingenierías, 16(2), 71-77 (2017)

Ugartemendia, M., Manual Técnicas de Lectura (2018)

Van Geffen, P., J. Reenalda, P.H. Veltink y B.F.J.M. Koopman, Decoupled Pelvis Adjustment to Induce Lumbar Motion: A Technique that Controls Low Back Load in Sitting, https://doi.org/10.1016/j.ergon.2009.08.015, Int. J. Ind. Ergon, 40(1), 47-54 (2010)

Wainschenker, R., J. Doorn y M. Castro, Medición Cuantitativa de la Velocidad del Habla, http://journal.sepln.org/sepln/ojs/ojs/index.php/pln, ISSN: 1135-5948, Procesamiento del Lenguaje Natural, (28),99-104 (2002)

Werth, A. y K. Babski-Reeves, Effects of Portable Computing Devices on Posture, Muscle Activation Levels and Efficiency, doi: 10.1016/j.apergo.2014.05.008, Appl Ergon, 45(6), 1603-1609 (2014)

Yeowa, P. y R. Sen, Productivity and Quality Improvements, Revenue Increment, and Rejection Cost Reduction in the Manual Component Insertion Lines Through the Application of Ergonomics, https://doi.org/10.1016/j.ergon.2005.12.008, Int. J. Ind. Ergon, 36(4), 367-377 (2006) 